\title{
ASEXUAL VERSUS PROMISCUOUS BISEXUAL GALTON-WATSON PROCESSES: THE EXTINCTION PROBABILITY RATIO
}

\author{
By Gerold Alsmeyer And Uwe RöSler \\ Westfälische Wilhelms-Universität Münster and \\ Christian-Albrechts-Universität Kiel
}

\begin{abstract}
We consider the supercritical bisexual Galton-Watson process (BGWP) with promiscuous mating, that is, a branching process which behaves like an ordinary supercritical Galton-Watson process (GWP) as long as at least one male is born in each generation. For a certain example, it was pointed out by Daley, Hull and Taylor [J. Appl. Probab. 23 (1986) 585-600] that the extinction probability of such a BGWP apparently behaves like a constant times the respective probability of its asexual counterpart (where males do not matter) if the number of ancestors grows to $\infty$. In an earlier paper, we provided general upper and lower bounds for the ratio between both extinction probabilities and also numerical results that seemed to confirm the convergence of that ratio. However, theoretical considerations rather led us to the conjecture that this does not generally hold. The present article turns this conjecture into a rigorous result. The key step in our analysis is to identify the extinction probability ratio as a certain functional of a subcritical ordinary GWP and to prove its continuity as a function of the number of ancestors in a suitable topology associated with the entrance Martin boundary of that GWP.
\end{abstract}

1. Introduction and main results. The bisexual Galton-Watson process with promiscuous mating $\left(\mathcal{Z}_{n}\right)_{n \geq 0}$, shortly called promiscuous BGWP, is defined as follows: consider a two-sex population process $\left(\mathcal{Z}_{n}^{\mathrm{F}}, \mathcal{Z}_{n}^{\mathrm{M}}\right)_{n \geq 0}$ whose $n$th generation consists of $\mathcal{Z}_{n}^{\mathrm{F}}$ females and $\mathcal{Z}_{n}^{\mathrm{M}}$ males. Females within one generation reproduce according to an ordinary two-type Galton-Watson process (GWP) with product reproduction law $\mathbf{p}^{\mathrm{F}} \otimes \mathbf{p}^{\mathrm{M}}$ as long as at least one male is alive. Plainly, $\mathbf{p}^{\mathrm{F}}=\left(p_{j}^{\mathrm{F}}\right)_{j \geq 0}$ and $\mathbf{p}^{\mathrm{M}}=\left(p_{j}^{\mathrm{M}}\right)_{j \geq 0}$ describe the number of female, respectively, male offspring. We are therefore given

$$
Z_{n} \stackrel{\text { def }}{=} \mathcal{Z}_{n}^{\mathrm{F}} \mathbb{1}_{(0, \infty)}\left(\mathcal{Z}_{n}^{\mathrm{M}}\right)
$$

mating units in the $n$th generation, the pertinent mating function being $\zeta(x, \cdot)=$ $x \mathbb{1}_{(0, \infty)}$. The formal definition of $\left(\mathcal{Z}_{n}^{\mathrm{F}}, \mathcal{Z}_{n}^{\mathrm{M}}\right)_{n \geq 0}$ thus takes the form

$$
\left(\mathcal{Z}_{n+1}^{\mathrm{F}}, \mathcal{Z}_{n+1}^{\mathrm{M}}\right)=\sum_{j=1}^{Z_{n}}\left(\xi_{n, j}, \eta_{n, j}\right) \text {, }
$$

Received December 2000; revised June 2001.

AMS 2000 subject classifications. Primary 60J80; secondary $60 \mathrm{~J} 45$.

Key words and phrases. Bisexual Galton-Watson process, promiscuous mating, extinction probability, quasi-invariant measure, time reversal. 
with i.i.d. random vectors $\left(\xi_{n, j}, \eta_{n, j}\right), n \geq 0, j \geq 1$, with common distribution $\mathbf{p}^{\mathrm{F}} \otimes \mathbf{p}^{\mathrm{M}}$.

Bisexual GWP's with various mating functions were introduced by Daley [6] and further investigated in [5] and [7-9]. The present article is a continuation of [1] where we compared in some detail the extinctive behavior of a promiscuous BGWP $\left(Z_{n}\right)_{n \geq 0}$ with that of its asexual counterpart, henceforth denoted by $\left(F_{n}\right)_{n \geq 0}$. Let $P_{j}$ be such that $P_{j}\left(\mathcal{Z}_{0}=F_{0}=j\right)=1$ for each $j \geq 1$ and define the extinction probability function

$$
\mathbf{q}(j) \stackrel{\text { def }}{=} P_{j}\left(\mathcal{Z}_{n}=0 \text { for some } n \geq 0\right), \quad j \in \mathbb{N}_{0},
$$

pertaining to $\left(\mathcal{Z}_{n}\right)_{n \geq 0}$. Plainly, the reproduction law of the ordinary GWP $\left(F_{n}\right)_{n \geq 0}$ is $\mathbf{p}^{\mathrm{F}}$, its extinction probability function $q^{j}$ for some $q \in[0,1]$. We are interested in the supercritical case when $\mathbf{q}(j)<1$ for all $j \geq 1$, a standing assumption hereafter. For the promiscuous BGWP this is easily seen to be equivalent to $p_{0}^{\mathrm{M}}<1$ and $\mu \stackrel{\text { def }}{=} \sum_{j \geq 1} j p_{j}^{\mathrm{F}}>1$. Hence $\left(F_{n}\right)_{n \geq 0}$ is also supercritical and its extinction probability $q$ less than 1 . A numerical study by Daley, Hull and Taylor [7] showed for the case where $\mathbf{p}^{\mathrm{F}}$ and $\mathbf{p}^{\mathrm{M}}$ are Poisson with mean 1.2 that the extinction probability ratio

$$
\mathbf{r}(k) \stackrel{\text { def }}{=} \frac{\mathbf{q}(k)}{q^{k}}, \quad k \in \mathbb{N},
$$

apparently converges very rapidly to approximately 1.33 . On the other hand, they had no theoretical justification for this phenomenon and our analysis in [1] indeed showed that this can neither be given shortly nor by easy arguments. Let $\hat{P}_{k}=P_{k}\left(\cdot \mid F_{n} \rightarrow 0\right)$ with expectation operator $\hat{E}_{k}$ and put $\kappa \stackrel{\text { def }}{=} p_{0}^{\mathrm{M}}$. By exploiting a functional equation for $\mathbf{r}(k)$, namely,

$$
\mathbf{r}(k)=\left(\frac{\kappa}{q}\right)^{k}+\left(1-\kappa^{k}\right) \hat{E}_{k} \mathbf{r}\left(F_{1}\right)
$$

for each $k \geq 0$, we were led in [1] to lower and upper bounds for $\mathbf{r}(k)$, depending on the model parameters. Numerical studies for various sets of parameters further confirmed the observation of Daley, Hull and Taylor that $\mathbf{r}(k)$ rapidly stabilizes for increasing $k$ if $\kappa<q$. However, based on arguments beyond the scope of that article, we conjectured that $\mathbf{r}(k)$ may actually not always converge but oscillate very slowly, a "near-constancy" phenomenon also encountered for the so-called Harris function of certain supercritical ordinary GWP's; see, for example, [4]. The main result of this article, Theorem 2.1, shows that this conjecture is correct. The proof is based on potential theory for subcritical GWP's which is therefore shortly reviewed from [3] in Section 3.

Iterating (1.2) leads to the fundamental identity (see (3.12) in [1])

$$
\mathbf{r}(k)=\left(\frac{\kappa}{q}\right)^{k}+\hat{E}_{k}\left(\sum_{j=1}^{\tau}\left(\frac{\kappa}{q}\right)^{F_{j}} \prod_{i=0}^{j-1}\left(1-\kappa^{F_{i}}\right)\right),
$$


where $\tau$ denotes the extinction time of $\left(F_{n}\right)_{n \geq 0}$. Note that, under $\hat{P}_{k},\left(F_{n}\right)_{n \geq 0}$ forms an ordinary subcritical GWP with $k$ ancestors, reproduction mean $\hat{\mu}=$ $f^{\prime}(q)$, offspring distribution $\hat{p}^{\mathrm{F}}=\left(q^{j-1} p_{j}^{\mathrm{F}}\right)_{j \geq 0}$ and offspring generating function $\hat{f}(s)=q^{-1} f(s q)$, where $f$ is the generating function of $\mathbf{p}^{\mathrm{F}}$; see [3], page 37. Note that

$$
\hat{P}_{1}\left(F_{1}>1\right)=\sum_{j \geq 2} q^{j-1} p_{j}^{\mathrm{F}}>0
$$

and that $q<1$ clearly implies the $(X \log X)$-condition for $\left(F_{n}\right)_{n \geq 0}$ under the $\hat{P}_{k}$, that is,

$$
\hat{E}_{k} F_{1} \log F_{1}<\infty \text {. }
$$

Our main concern hereafter will be the case $0<\kappa<q$ where the nearconstancy phenomenon turns up, but we will also provide a result for the case $\kappa=q$ (Theorem 2.2). If $\kappa>q$, we already gave a satisfactory answer in [1], Corollary 3.2, which states that $\kappa^{-k} \mathbf{q}(k)$ converges to 1 at an exponential rate.

2. Main results. A look at identity (1.3) shows that its further investigation no longer requires dealing with the original model of a promiscuous BGWP from which it came out. We may rather adopt the viewpoint of dealing with a certain functional in two arguments, $\kappa$ and $q$, of an ordinary subcritical GWP. We will therefore simplify our previous notation and use the one for Galton-Watson branching processes in [3] to which we will frequently refer. So from now on let $\left(Z_{n}\right)_{n \geq 0}$ be a subcritical GWP with offspring distribution $\left(p_{j}\right)_{j \geq 0}$, offspring generating function $f(s)=\sum_{j \geq 0} p_{j} s^{j}$, reproduction mean $\mu=f^{\prime}(1)<1$ and extinction time $\tau$. Notice that now $f(q) \neq q$. For each $k \geq 1, P_{k}$ shall denote the probability measure under which $Z_{0}=k$. If $k=1$, we also write $P$ instead of $P_{1}$. We further assume [see also (1.4) and (1.5)]

$$
p_{1}>0, \quad p_{0}+p_{1}<1
$$

and the $(X \log X)$-condition

$$
E Z_{1} \log Z_{1}<\infty
$$

These conditions will in fact be needed in the course of our subsequent analysis. The first condition together with $p_{0}>0$ ensures that all states $i \geq 1$ are communicating for $\left(Z_{n}\right)_{n \geq 0}$ and, as a consequence, that all quasi-invariant measures (see Section 3 ) have positive mass at each $i \geq 1$.

The function $\mathbf{r}(k)=\mathbf{r}(\kappa, q, k)$ now clearly takes the form

$$
\mathbf{r}(k)=\left(\frac{\kappa}{q}\right)^{k}+E_{k}\left(\sum_{j=1}^{\tau}\left(\frac{\kappa}{q}\right)^{Z_{j}} \prod_{i=0}^{j-1}\left(1-\kappa^{Z_{i}}\right)\right)
$$


for $k \in \mathbb{N}$ and $0<\kappa \leq q<1$. Since $\mathbf{r}(k)$ is also a functional of $\left(Z_{\tau-n}\right)_{0 \leq n \leq \tau}$ under $P_{k}$, its asymptotic behavior, as $k \rightarrow \infty$, should be linked to the limit behavior of $\left(Z_{\tau-n}\right)_{0 \leq n \leq \tau}$ under $P_{k}$. Unfortunately, there is not just one limiting distribution but infinitely many, essentially the Martin entrance boundary of $\left(Z_{n}\right)_{n \geq 0}$. This comes out from potential theoretic considerations for subcritical GWP's as described, for example, in [3]. A short review of the most important facts from there will be given in the following section. Here we confine ourselves to a sketchy description in order to formulate our results.

Let $Q_{k}$ be the distribution of the time reversal $\left(Z_{\tau-n}\right)_{0 \leq n \leq \tau}$ under $P_{k}$. Any probability measure $Q$ in the closure of $\left\{Q_{k}, k \geq 1\right\}$ with respect to weak convergence defines a Markov chain $\left(W_{n}\right)_{n \geq 0}$ on $\mathbb{N}_{0}$ with transition matrix $\left(q_{i j}\right)_{i, j \geq 0}$, say, and corresponds uniquely to a quasi-invariant measure $\eta=\left(\eta_{i}\right)_{i \geq 1}$ for $\left(Z_{n}\right)_{n \geq 0}$ (see Section 3) via the relation

$$
q_{i j}= \begin{cases}0, & \text { if } i=j=0, \\ \eta_{j} p_{0}^{j}, & \text { if } i=0, j \geq 1, \\ \eta_{i} p_{i j} / \eta_{j}, & \text { if } i, j \geq 1,\end{cases}
$$

where $\eta$ is normalized such that $\sum_{j \geq 1} \eta_{j} p_{0}^{j}=1$. In our setting, we are interested in sequences $k_{n}, n \geq 1$, approaching $\infty$ in such a way that $\mathbf{r}\left(k_{n}\right)$ converges, as $n \rightarrow \infty$. It suffices to consider sequences $k_{n}, n \geq 1$, such that $Q_{k_{n}}$ converges weakly to some probability measure $Q$. We may identify $Q$ with a quasi-invariant measure $\eta$ via (2.4). As shown in [2], these are exactly the extremal elements in the convex set of all quasi-invariant measures (normalized as above), for which the circle forms a natural parametrization. We thus identify the closure of $\left\{Q_{k}, k \geq 1\right\}$ with the set $\mathfrak{N} \stackrel{\text { def }}{=} \mathbb{N} \cup(-1,0]$. The Martin topology on $\mathfrak{N}$, rendering weak continuity of $x \mapsto Q_{x}$, is isomorphic to the topology generated by the metric $\rho$ defined in Section 3. Taking these facts for granted, assertion (2.5) of Theorem 2.1 below should no longer be too surprising.

ThEOREM 2.1. Assume (2.1), (2.2) and $0<\kappa<q$. Then, for all $x \in(-1,0]$,

$$
\lim _{k \rightarrow \infty} \mathbf{r}(k)=\mathbf{r}(x) \stackrel{\text { def }}{=} E\left(\sum_{n \geq 0}\left(\frac{\kappa}{q}\right)^{W_{n}(x)} \prod_{i>n}\left(1-\kappa^{W_{i}(x)}\right) \mid W_{0}(x)=0\right),
$$

where $\left(W_{n}(x)\right)_{n \geq 0}$ is a Markov chain on $\mathbb{N}_{0}$ with distribution $Q_{x}$. Moreover, for each $q \in(0,1)$, there exist infinitely many $\kappa \in(0, q)$ such that $\mathbf{r}$ is not a constant.

THEOREM 2.2. Assume (2.1), (2.2) and $\kappa=q$ and put $a_{k} \stackrel{\text { def }}{=} E_{k} \tau$. Then

$$
\lim _{k \rightarrow \infty} \frac{\mathbf{q}(k)}{a_{k} q^{k}}=1 .
$$


3. Quasi-invariant measures and time reversal. We begin with a review of some basic facts from potential theory for subcritical GWP's as described in [3], Chapter 2. The notation is kept from there as far as possible. So let $\left(Z_{n}\right)_{n \geq 0}$ be an ordinary subcritical GWP with reproduction distribution $\left(p_{j}\right)_{j \geq 0}$ and reproduction mean $\mu=\sum_{j \geq 0} j p_{j}<1$. Let $f$ be the generating function of $\left(p_{j}\right)_{j \geq 0}$, that is, $f(s)=\sum_{j \geq 0} p_{j} s^{j}$, and let $f_{n}$ be its $n$-fold iterate.

Denote by $p_{i j}$ the transition probabilities of $\left(Z_{n}\right)_{n \geq 0}$. A $\sigma$-finite measure $\eta=\left(\eta_{j}\right)_{j \geq 1}$ on $\mathbb{N}$ is called quasi-invariant or quasi-stationary for $\left(Z_{n}\right)_{n \geq 0}$ if

$$
\eta_{j}=\sum_{i \geq 1} \eta_{i} p_{i j}
$$

for all $j \in \mathbb{N}$. Notice that we exclude the absorbing state 0 in the summation. The generating function $U(s)=\sum_{j \geq 1} \eta_{j} s^{j}$ of any such $\eta$ is analytic for $|s|<1$ and, if normalized so that $U\left(p_{0}\right)=1$, satisfies the functional relation

$$
1+U(s)=U(f(s)) .
$$

Conversely, this relation characterizes quasi-invariant measures ([3], Theorem 2.2). Since all states $i \geq 1$ communicate and $\eta_{j}=\sum_{i \geq 1} \eta_{i} p_{i j}^{(n)}$ for all $n \geq 1$, we infer $\eta_{i}>0$ for all $i \geq 1$, as already mentioned in Section 2 .

To describe all quasi-invariant measures for $\left(Z_{n}\right)_{n \geq 0}$, let ([3], Section 2.2, equation (3))

$$
U(s, t) \stackrel{\text { def }}{=} \sum_{n \in \mathbb{Z}}\left(\exp \left(Q(s) \mu^{n-t}\right)-\exp \left(Q(0) \mu^{n-t}\right)\right), \quad|s|<1, t \in(-1,0] .
$$

Here

$$
Q(s) \stackrel{\text { def }}{=} \lim _{n \rightarrow \infty} \mu^{-n}\left(f_{n}(s)-1\right), \quad s \in[0,1] .
$$

Note that $Q(f(s))=\mu Q(s)$ ([3], equation (10), page 40) and $Q(s)=Q(0)(1-$ $B(s)$ ) ([3], equation (30), page 47), where $B$ is the generating function

$$
B(s) \stackrel{\text { def }}{=} \sum_{j \geq 1} b_{j} s^{j}, \quad b_{j}=\lim _{n \rightarrow \infty} P\left(Z_{n}=j \mid Z_{n}>0\right) .
$$

The following result is shown in [2].

THEOREM 3.1. If $E Z_{1} \log Z_{1}<\infty$, then the space of quasi-invariant measures (up to positive scalars) is isomorphic to the set of probability measures on the circle. The bijection $\eta \leftrightarrow v$ can be stated as

$$
U_{\eta}=\int_{(-1,0]} U(\cdot, t) v(d t),
$$

where $U_{\eta}$ is the generating function of $\eta$. 
Recall that $\mathfrak{N}=\mathbb{N} \cup(-1,0]$. Define the map $\varphi: \mathfrak{N} \rightarrow \mathbb{C}$ by

$$
\varphi(x) \stackrel{\text { def }}{=} \begin{cases}\frac{x}{1+x} e^{2 \pi i \log _{\mu} x}, & \text { if } x \in \mathbb{N}, \\ e^{2 \pi i x}, & \text { if } x \in(-1,0]\end{cases}
$$

and then the metric $\rho$ on $\mathfrak{N}$ by

$$
\rho(x, y)=|\varphi(x)-\varphi(y)| .
$$

Notice that under this metric the closure of $\mathbb{N}$ is $(-1,0]$ and that $(-1,0]$ is endowed with the spherical topology. The latter is not true for the metric given in [3], page 69. An integer sequence $\left(k_{n}\right)_{n \geq 1}$ converges in the $\rho$-metric iff $\left(\mathbb{K}\left(k_{n}, \cdot\right)\right)_{n \geq 1}$ converges pointwise on $\mathbb{N}_{0}$, where

$$
\mathbb{K}(i, j) \stackrel{\text { def }}{=} \frac{\mathbb{G}(i, j)}{\sum_{k \geq 1} \mathbb{G}(i, k) p_{0}^{k}}
$$

is the Martin kernel and

$$
\mathbb{G}(i, j) \stackrel{\text { def }}{=} \sum_{n \geq 0} p_{i j}^{(n)}
$$

is the Green kernel. Every such sequence $\left(k_{n}\right)_{n \geq 0}$ with a $\rho$-limit $t \in(-1,0]$ will be called a Martin sequence hereafter, and we write $k_{n} \stackrel{\rho}{\rightarrow} t$ [equivalent is $\left.\varphi\left(k_{n}\right) \rightarrow \varphi(t)\right]$. The closure of $\{\mathbb{K}(\cdot, j), j \in \mathbb{N}\}$ is isomorphic to $(\mathfrak{N}, \rho)$. For such a Martin sequence we further have

$$
\lim _{n \rightarrow \infty} \mathbb{K}\left(k_{n}, j\right)=\lim _{n \rightarrow \infty} \mathbb{G}\left(k_{n}, j\right)=\eta_{j}(t),
$$

where $\eta(t)=\left(\eta_{j}(t)\right)_{j \geq 1}$ is the quasi-invariant measure with generating function $U(\cdot, t), t \in(-1,0]$ as defined above. For the first equality it should be noticed that

$$
\begin{aligned}
\sum_{l \geq 1} \mathbb{G}\left(k_{n}, l\right) p_{0}^{l} & =\sum_{m \geq 1}\left(f_{m}^{k_{n}}\left(p_{0}\right)-f_{m}^{k_{n}}(0)\right) \\
& =\sum_{m \geq 1}\left(f_{m+1}^{k_{n}}(0)-f_{m}^{k_{n}}(0)\right)=1-f^{k_{n}}(0),
\end{aligned}
$$

which converges to 1 as $n \rightarrow \infty$. Note also that $\eta(t)$ is continuous in $t$; see [3], page 69.

The time reversal $\left(W_{n}(t)\right)_{n \geq 0}$, say, of $\left(Z_{n}\right)_{n \geq 0}$ with respect to any quasiinvariant measure $\eta(t)$ is a Markov chain with $n$-step transition matrix $\mathbf{Q}^{n}(t)=$ $\left(q_{i j}^{(n)}(t)\right)_{i, j \geq 0}, n \geq 1$, where

$$
q_{i j}^{(n)}(t) \stackrel{\text { def }}{=} \begin{cases}0, & \text { if } i=j=0, \\ \eta_{j}(t) P_{j}(\tau=n), & \text { if } i=0, j \geq 1, \\ \frac{\eta_{j}(t) p_{j i}^{(n)}}{\eta_{i}(t)}, & \text { if } i, j \geq 1 .\end{cases}
$$


The associated Green function is denoted $H(i, j, t)=\sum_{n \geq 0} q_{i j}^{(n)}(t)$ and satisfies $H(0,0, t)=1, H(0, j, t)=\eta_{j}(t)$ for $j \geq 1$ and $H(i, j, t)=\eta_{j}(t) \mathbb{G}(j, i) / \eta_{i}(t)$, otherwise.

LEMmA 3.2. For any $i_{1}, \ldots, i_{m} \in \mathbb{N}$ and $m \in \mathbb{N}$, the function $f_{i_{1}, \ldots, i_{m}}$ : $\mathbb{N} \cup(-1,0] \rightarrow[0,1]$,

$$
f_{i_{1}, \ldots, i_{m}}(t) \stackrel{\text { def }}{=} \begin{cases}P_{t}\left(Z_{\tau-m}=i_{m}, \ldots, Z_{\tau-1}=i_{1}, Z_{\tau}=0\right), & \text { if } t \in \mathbb{N}, \\ P\left(W_{1}(t)=i_{1}, \ldots, W_{m}(t)=i_{m} \mid W_{0}(t)=0\right), & \text { if } t \in(-1,0],\end{cases}
$$

is continuous in the $\rho$-metric.

ProOF. Let first $\mathbb{N} \ni k_{n} \stackrel{\rho}{\rightarrow} t \in(-1,0]$ be a Martin sequence. Then, as $n \rightarrow \infty$,

$$
\begin{aligned}
f_{i_{1}, \ldots, i_{m}}\left(k_{n}\right) & =P_{k_{n}}\left(Z_{\tau-m}=i_{m}, \ldots, Z_{\tau-1}=i_{1}, Z_{\tau}=0\right) \\
& =\sum_{l \geq 0} P_{k_{n}}\left(Z_{l}=i_{m}\right) p_{i_{m} i_{m-1}} \cdots p_{i_{1} 0} \\
& =\mathbb{G}\left(k_{n}, i_{m}\right) \frac{1}{\eta_{i_{m}}(t)} q_{i_{m-1} i_{m}}(t) \cdots q_{0 i_{1}}(t) \\
& \rightarrow q_{i_{m-1} i_{m}}(t) \cdots q_{0 i_{1}}(t)=f_{i_{1}, \ldots, i_{m}}(t) .
\end{aligned}
$$

For a sequence $(-1,0] \ni t_{n} \stackrel{\rho}{\rightarrow} t \in(-1,0]$, the assertion follows from the continuity of the $\eta_{j}(t)$ in $t$.

Notice that Lemma 3.2 states, in particular, that, for every Martin sequence $k_{n} \stackrel{\rho}{\rightarrow} t$,

$$
\begin{aligned}
& \lim _{n \rightarrow \infty} P_{k_{n}}\left(Z_{\tau-m}=i_{m}, \ldots, Z_{\tau-1}=i_{1}, Z_{\tau}=0\right) \\
& \quad=P\left(W_{1}(t)=i_{1}, \ldots, W_{m}(t)=i_{m} \mid W_{0}(t)=0\right)
\end{aligned}
$$

for all $i_{1}, \ldots, i_{m} \in \mathbb{N}$ and $m \in \mathbb{N}$ which explains the meaning of $\left(W_{n}(t)\right)_{n \geq 0}$ as a time reversal of $\left(Z_{n}\right)_{n \geq 0}$.

4. Proof of Theorem 2.1. Let $R: \mathfrak{N} \times[0,1) \times[0,1) \rightarrow[0, \infty]$ be the function defined by

$$
R(x, u, v)= \begin{cases}E_{x}\left(\sum_{n=0}^{\tau} u^{Z_{n}} \prod_{i=0}^{n-1}\left(1-v^{Z_{i}}\right)\right), & \text { if } x \in \mathbb{N}, \\ E\left(\sum_{n \geq 0} u^{W_{n}(x)} \prod_{i>n}\left(1-v^{W_{i}(x)}\right) \mid W_{0}(x)=0\right), & \text { if } x \in(-1,0] .\end{cases}
$$

We will prove the following important fact in this section. 
Proposition 4.1. The function $R$ is finite and continuous in the product topology induced by $(\mathfrak{N}, \rho) \otimes\left([0,1)^{2}\right.$, Euclidean $)$.

The proof of this result is provided through a series of lemmas. Fix $N \in \mathbb{N}$ and define

$$
R_{N}(x, u, v)= \begin{cases}E_{x}\left(\sum_{n=\tau-N}^{\tau} u^{Z_{n}} \prod_{i=\tau-N}^{n-1}\left(1-v^{Z_{i}}\right)\right), & \text { if } x \in \mathbb{N}, \\ E\left(\sum_{n=0}^{N} u^{W_{n}(x)} \prod_{i=n+1}^{N}\left(1-v^{W_{i}(x)}\right) \mid W_{0}(x)=0\right), & \text { if } x \in(-1,0] .\end{cases}
$$

Our program is to show first that $R_{N}$ is continuous for each $N$ (Lemma 4.2) and then in several steps that $R-R_{N}$ converges to 0 uniformly on compact sets (Lemmas 4.3-4.5). This clearly implies the asserted continuity of $R$.

LEMMA 4.2. For each $N \in \mathbb{N}$, the function $R_{N}$ is continuous in the product topology induced by $(\mathfrak{N}, \rho) \otimes\left([0,1)^{2}\right.$, Euclidean $)$.

PROOF. Fix $N \in \mathbb{N}$, take a sequence $\left(x_{n}, u_{n}, v_{n}\right)$ convergent to $(x, u, v)$ and write

$$
\begin{aligned}
& \left|R_{N}\left(x_{n}, u_{n}, v_{n}\right)-R_{N}(x, u, v)\right| \\
& \quad \leq\left|R_{N}\left(x_{n}, u_{n}, v_{n}\right)-R_{N}\left(x_{n}, u, v\right)\right|+\left|R_{N}\left(x_{n}, u, v\right)-R_{N}(x, u, v)\right| .
\end{aligned}
$$

The second expression on the right-hand side tends to 0 by an application of Lemma 3.1 because $R_{N}(\cdot, u, v)$ is the expectation of a bounded function w.r.t. the weakly convergent discrete probability distributions $P_{x}\left(\left(Z_{\tau-N}, \ldots, Z_{\tau}\right) \in \cdot\right)$. As for the first, it is easy to show uniform convergence in $x_{n}$. Indeed, if $\left|u^{\prime}-u\right|<\varepsilon$ and $\left|v-v^{\prime}\right|<\varepsilon$, then

$$
\begin{gathered}
\left|R_{N}\left(x, u^{\prime}, v^{\prime}\right)-R_{N}(x, u, v)\right| \leq E_{x}\left(\sum_{n=\tau-N}^{\tau}(u+\varepsilon)^{Z_{n}} \prod_{i=\tau-N}^{n-1}\left(1-(v-\varepsilon)^{Z_{i}}\right)\right. \\
\left.-(u-\varepsilon)^{Z_{n}} \prod_{i=\tau-N}^{n-1}\left(1-(v+\varepsilon)^{Z_{i}}\right)\right) \\
=E_{x} \Delta_{\varepsilon, u, v}\left(Z_{\tau-N}, \ldots, Z_{\tau}\right),
\end{gathered}
$$


where $\Delta_{\varepsilon, u, v}$ is a bounded function defined in the obvious manner. Notice that $\Delta_{\varepsilon, u, v} \downarrow 0$ as $\varepsilon \downarrow 0$. Hence, by another appeal to Lemma 3.1 and the monotone convergence theorem,

$$
\begin{aligned}
\lim _{n \rightarrow \infty} & \left|R_{N}\left(x_{n}, u_{n}, v_{n}\right)-R_{N}\left(x_{n}, u, v\right)\right| \\
& \leq \lim _{\varepsilon \downarrow 0} \lim _{n \rightarrow \infty} E_{x_{n}} \Delta_{\varepsilon, u, v}\left(Z_{\tau-N}, \ldots, Z_{\tau}\right) \\
& =\lim _{\varepsilon \downarrow 0} E\left(\Delta_{\varepsilon, u, v}\left(W_{N}(x), \ldots, W_{0}(x)\right) \mid W_{0}(x)=0\right)=0 .
\end{aligned}
$$

To show uniform compact convergence of $R_{N}$ to $R$, as $N \rightarrow \infty$, we first observe that, for $k \in \mathbb{N}$,

$$
\begin{aligned}
\left|R(k, u, v)-R_{N}(k, u, v)\right| \\
\leq E_{k}\left(\sum_{n=0}^{\tau-N-1} u^{Z_{n}} \prod_{i=0}^{n-1}\left(1-v^{Z_{i}}\right)\right) \\
\quad+E_{k}\left(\sum_{n=\tau-N}^{\tau} u^{Z_{n}} \prod_{i=\tau-N}^{n-1}\left(1-v^{Z_{i}}\right)\left(1-\prod_{i=0}^{\tau-N-1}\left(1-v^{Z_{i}}\right)\right)\right) \\
\leq E_{k}\left(\sum_{n=0}^{\tau-N-1} u^{Z_{n}}\right)+N E_{k}\left(1-\prod_{i=0}^{\tau-N-1}\left(1-v^{Z_{i}}\right)\right) \\
\leq N E_{k}\left(\sum_{n=0}^{\tau-N-1}\left(u^{Z_{n}}+v^{Z_{n}}\right)\right) \\
\leq 2 N E_{k}\left(\sum_{n=0}^{\tau-N-1}(u \vee v)^{Z_{n}}\right),
\end{aligned}
$$

where

$$
1-\prod_{i=0}^{n}\left(1-c_{i}\right) \leq \sum_{i=0}^{n} c_{i}
$$

for $c_{0}, \ldots, c_{n} \in[0,1]$, has been used for the penultimate inequality. In view of the subsequent estimations, we note that (4.2) remains true if $n=\infty$. For $x \in(-1,0]$, we further have 


$$
\begin{aligned}
& \left|R(x, u, v)-R_{N}(x, u, v)\right| \\
& \leq \sum_{n>N} E\left(u^{W_{n}(x)} \prod_{i>n}\left(1-v^{W_{i}(x)}\right) \mid W_{0}(x)=0\right) \\
& \quad+\sum_{n=0}^{N} E\left(u^{W_{n}(x)} \prod_{i=n+1}^{N}\left(1-v^{W_{i}(x)}\right)\left(1-\prod_{j>N}\left(1-v^{W_{j}(x)}\right)\right) \mid W_{0}(x)=0\right) \\
& \leq \sum_{n>N} E\left(u^{W_{n}(x)} \mid W_{0}(x)=0\right)+N E\left(1-\prod_{j>N}\left(1-v^{W_{j}(x)}\right) \mid W_{0}(x)=0\right) \\
& \leq 2 N \sum_{n>N} E\left((u \vee v)^{W_{n}(x)} \mid W_{0}(x)=0\right) .
\end{aligned}
$$

Since the latter inequality is easier to handle we first show the following result.

LEMMA 4.3. For all $y, w<1$,

$$
\lim _{N \rightarrow \infty} \sup _{x \leq y ; u, v \leq w}\left|R(x, u, v)-R_{N}(x, u, v)\right|=0 .
$$

PROOF. Recalling from (3.2) the definition of $q_{0 i}^{(n)}$, we obtain

$$
\begin{aligned}
& N \sum_{n>N} E\left((u \vee v)^{W_{n}(x)} \mid W_{0}(x)=0\right) \\
& \leq N \sum_{n>N} E\left(w^{W_{n}(x)} \mid W_{0}(x)=0\right) \\
& =N \sum_{n>N} \sum_{i \geq 1} w^{i} q_{0 i}^{(n)} \\
& =N \sum_{n>N} \sum_{i \geq 1} w^{i} \eta_{i}(x) P_{i}(\tau=n) \\
& =N \sum_{i \geq 1} w^{i} \eta_{i}(x) P_{i}(\tau>N) \\
& =N \sum_{i \geq 1} w^{i} \eta_{i}(x) P_{i}\left(Z_{N}>0\right) \\
& =N \sum_{i \geq 1} w^{i} \eta_{i}(x)\left(1-f_{N}^{i}(0)\right) \\
& =N\left(U(w, x)-U\left(w f_{N}(0), x\right)\right) \\
& \leq C(w, y) N w\left(1-f_{N}(0)\right),
\end{aligned}
$$


where $C(w, y) \stackrel{\text { def }}{=} \max _{x \leq y ; u \leq w} D_{u} U(u, x)<\infty$ as one can easily check. The assertion now follows because $N\left(1-f_{N}(0)\right) \rightarrow 0$ as $N \rightarrow \infty$; see [3], Section 1.11 .

To further exploit (4.1) for our purposes, we have to consider the functions

$$
\begin{aligned}
g_{N}(k, u) & \stackrel{\text { def }}{=} E_{k}\left(\sum_{n=0}^{\tau-N-1} u^{Z_{n}}\right), \\
h(k, u) & \stackrel{\text { def }}{=} \sum_{n \geq 0} E_{k} u^{Z_{n}} \mathbb{1}_{\left\{Z_{n}>0\right\}}=\sum_{n \geq 0}\left(f_{n}^{k}(u)-f_{n}^{k}(0)\right),
\end{aligned}
$$

$k, N \in \mathbb{N}, u \in[0,1)$, which are related as follows. Let $\left(Z_{n}^{\prime}\right)_{n \geq 0}$ be an independent copy of $\left(Z_{n}\right)_{n \geq 0}$ with $Z_{0}=Z_{0}^{\prime}$ under each $P_{i}$.

$$
\begin{aligned}
g_{N}(k, u) & =E_{k}\left(\sum_{n \geq 0} u^{Z_{n}} \mathbb{1}_{\{\tau>n+N\}}\right)=\sum_{n \geq 0} E_{k} u^{Z_{n}} \mathbb{1}_{\left\{Z_{n+N}>0\right\}} \\
& =\sum_{n \geq 0} E_{k} u^{Z_{n}} P_{Z_{n}}\left(Z_{N}^{\prime}>0\right)=\sum_{n \geq 0} E_{k} u^{Z_{n}}\left(1-f_{N}^{Z_{n}}(0)\right) \\
& =\sum_{n \geq 0}\left(f_{n}^{k}(u)-f_{n}^{k}\left(u f_{N}(0)\right)\right)=h(k, u)-h\left(k, u f_{N}(0)\right) .
\end{aligned}
$$

\section{LEMMA 4.4. The function $h$ satisfies}

$$
\sup _{k \in \mathbb{N}} h(k, u) \leq m(u)
$$

for each $u<1$, where $m(u) \stackrel{\text { def }}{=} \inf \left\{n \geq 1: f_{n}(0) \geq u\right\}$. Furthermore,

$$
\sup _{k \geq 1 ; u, v \leq w}|h(k, u)-h(k, v)|<m(w+\varepsilon)\left(2\left(\frac{w}{w+\varepsilon}\right)^{N}+\frac{|u-v|}{w} N\right)
$$

for all $\varepsilon>0,0<w<1-\varepsilon$ and $N \in \mathbb{N}$.

Proof. By using $0 \leq \sum_{n \geq 0}\left(1-f_{n}^{k}(u)\right)<\infty$ for all $k \in \mathbb{N}$ and $u<1$, we obtain, with $m=m(u)$,

$$
\begin{aligned}
h(k, u) & =\sum_{n \geq 0}\left(f_{n}^{k}(u)-1\right)-\sum_{n \geq m}\left(f_{n-m}^{k}\left(f_{m}(0)\right)-1\right)-\sum_{n=0}^{m-1}\left(f_{n}^{k}(0)-1\right) \\
& =\sum_{n \geq 0}\left(f_{n}^{k}(u)-f_{n}^{k}\left(f_{m}(0)\right)\right)+\sum_{n=0}^{m-1}\left(1-f_{n}^{k}(0)\right) \leq m
\end{aligned}
$$

because the first sum in the previous line is negative. 
To prove (4.6), we note first that

$$
\begin{aligned}
h(k, u) & =\sum_{n \geq 0} E_{k} u^{Z_{n}} \mathbb{1}_{\left\{Z_{n}>0\right\}} \\
& =\sum_{i \geq 1} u^{i} \sum_{n \geq 0} P_{k}\left(Z_{n}=i\right)=\sum_{i \geq 1} u^{i} \mathbb{G}(k, i) .
\end{aligned}
$$

Define $h_{N}(k, u) \stackrel{\text { def }}{=} \sum_{i=1}^{N} u^{i} \mathbb{G}(k, i)$ and choose an arbitrary $\varepsilon>0$, w.l.o.g. $<1-w$. Then, for all $k \in \mathbb{N}$ and $u \leq w$,

$$
\begin{aligned}
h(k, u)-h_{N}(k, u) & =\sum_{i>N} u^{i} \mathbb{G}(k, i) \leq\left(\frac{u}{w+\varepsilon}\right)^{N} \sum_{i>N}(w+\varepsilon)^{i} \mathbb{G}(k, i) \\
& =\left(\frac{u}{w+\varepsilon}\right)^{N} h(k, w+\varepsilon) \leq\left(\frac{w}{w+\varepsilon}\right)^{N} m(w+\varepsilon),
\end{aligned}
$$

where (4.5) has been used for the final inequality. Moreover, for all $u, v \leq w$ and $N \geq 1$,

$$
\begin{aligned}
\left|h_{N}(k, u)-h_{N}(k, v)\right| & =|u-v| \sum_{i=1}^{N}\left(\frac{u^{i}-v^{i}}{u-v}\right) \mathbb{G}(k, i) \\
& =|u-v| \sum_{i=1}^{N} \sum_{j=0}^{i-1} u^{j} v^{i-1-j} \mathbb{G}(k, i) \\
& \leq \frac{|u-v|}{w} \sum_{i=1}^{N} i w^{i-1} \mathbb{G}(k, i) \leq \frac{|u-v|}{w} N m(w),
\end{aligned}
$$

the last inequality again by (4.5). By combining (4.7) and (4.8) with a simple application of the triangle inequality, we finally obtain (4.6).

Going back to (4.1), we are now ready to prove the following result.

LEMMA 4.5. For all $w<1$,

$$
\lim _{N \rightarrow \infty} \sup _{k \geq 1 ; u, v \leq w}\left|R(k, u, v)-R_{N}(k, u, v)\right|=0 .
$$

PROOF. Indeed, we infer, with the help of (4.1), (4.4) and the previous lemma, that

$$
\begin{aligned}
& \limsup _{N \rightarrow \infty} \sup _{k \geq 1 ; u, v \leq w}\left|R(k, u, v)-R_{N}(k, u, v)\right| \\
& \quad \leq 2 \limsup _{N \rightarrow \infty} N \sup _{k \geq 1 ; u, v \leq w}\left|h(k, u \vee v)-h\left(k,(u \vee v) f_{N}(0)\right)\right| \\
& \quad \leq 2 m(w+\varepsilon) \limsup _{N \rightarrow \infty}\left(2 N\left(\frac{w}{w+\varepsilon}\right)^{N}+\frac{(u \vee v)\left(1-f_{N}(0)\right)}{w} N^{2}\right)=0,
\end{aligned}
$$

recalling that $1-f_{N}(0)$ converges to 0 exponentially fast; see [3], Section 1.11. 
ProOF OF PROPOSITION 4.1. A combination of Lemmas 4.3 and 4.5 clearly yields uniform compact convergence of the $R_{N}$ to $R$. Since the $R_{N}$ are further continuous by Lemma 4.2, we conclude the continuity of $R$ as claimed.

In view of the main assertion of Theorem 2.1, namely, the nonconstancy of the extinction probability ratio $\mathbf{r}(x)$ for suitable pairs $(\kappa, q)$, two further lemmas are needed.

LEMMA 4.6. The function $\eta_{1}(t), t \in(-1,0]$, is not a constant.

PROOF. Note first that

$$
\eta_{1}(t)=\left.D_{s} U(s, t)\right|_{s=0}=Q^{\prime}(0) \sum_{n \in \mathbb{Z}} \mu^{n-t} \exp \left(Q(0) \mu^{n-t}\right) .
$$

Since our assumptions in Section 2 guarantee $\eta_{1}$ to be everywhere positive, we particularly have $Q^{\prime}(0)>0$. We make the change of variables $x=\mu^{-t}$, that is, $t=-\log _{\mu} x$. Defining

$$
\Psi(x, y) \stackrel{\text { def }}{=} \sum_{n \in \mathbb{Z}}\left(e^{x y \mu^{n}}-e^{y \mu^{n}}\right), \quad y<0<x,
$$

we obviously have

$$
D_{x} \Psi(x, y)=\sum_{n \in \mathbb{Z}} y \mu^{n} e^{x y \mu^{n}}
$$

and therefore

$$
\eta_{1}\left(-\log _{\mu} x\right)=\frac{Q^{\prime}(0)}{Q(0)} x D_{x} \Psi(x, Q(0)) .
$$

Now suppose $\eta_{1}$ is constant and infer

$$
D_{x} \Psi(x, Q(0))=\frac{c}{x}
$$

for all $x \in[1,1 / \mu)$ and some constant $c<0$. The equality extends to all $x>0$ because both sides are evidently analytic functions on the half plane of complex numbers with positive real part. Integration together with $\Psi(1, \cdot) \equiv 0$ then implies

$$
\Psi(x, Q(0))=\int_{1}^{x} D_{z} \Psi(z, Q(0)) d z=c \log x
$$

for all $x>0$.

Next, the functional equation

$$
\Psi(x, y)=\Psi\left(\frac{x y}{z}, z\right)+\Psi\left(\frac{z}{y}, y\right)
$$


for all $y, z<0<x$ together with (4.9) leads to

$$
\Psi(x, y)=\Psi\left(\frac{x y}{Q(0)}, Q(0)\right)+\Psi\left(\frac{Q(0)}{y}, y\right)=c \log \left(\frac{x y}{Q(0)}\right)+\Psi\left(\frac{Q(0)}{y}, y\right)
$$

for all $y<0<x$.

For $x=1$, we get

$$
\Psi\left(\frac{Q(0)}{y}, y\right)=c \log \left(\frac{Q(0)}{y}\right)
$$

and thereby

$$
\Psi(x, y)=c \log x
$$

for all $y<0<x$. Rewriting this result for $U(s, t)$, we find that

$$
U(s, t)=\Psi\left(\frac{Q(s)}{Q(0)}, \mu^{-t} Q(0)\right)=c \log \left(\frac{Q(s)}{Q(0)}\right),
$$

which is impossible because the $U(\cdot, t)$ are pairwise distinct by Theorem 3.1.

LEMMA 4.7. Given any $q \in(0,1)$, the function $R(\cdot, u / q, u)$ is not constant for all sufficiently small $u \in(0, q)$.

Proof. The function $R(x, u / q, u)$ is analytic in $u$ and $R(\cdot, 0,0) \equiv 1$. Writing

$$
\begin{aligned}
R(x, u / q, u)= & E\left(\prod_{i \geq 1}\left(1-u^{W_{i}(x)}\right) \mid W_{0}(x)=0\right) \\
& +\frac{u}{q} E\left(\sum_{n \geq 1}\left(\frac{u}{q}\right)^{W_{n}(x)-1} \prod_{i>n}\left(1-u^{W_{i}(x)}\right) \mid W_{0}(x)=0\right)
\end{aligned}
$$

and noting that $W_{n}(x) \geq 1$ for all $x \in(-1,0]$ and $n \geq 1$, it is easily verified that

$$
\left.D_{u} R(x, u / q, u)\right|_{u=0}=\frac{1-q}{q} \eta_{1}(x) .
$$

Since $\eta_{1}(x)$ is not constant in $x$, the same holds true for $D_{u} R(x, u / q, u)$ at $u=0$. Consequently, picking two distinct values $x_{1}, x_{2} \in(-1,0]$ with $\left.D_{u} R\left(x_{1}, u / q, u\right)\right|_{u=0} \neq\left. D_{u} R\left(x_{2}, u / q, u\right)\right|_{u=0}$, we must also have $R\left(x_{1}, u / q, u\right) \neq$ $R\left(x_{2}, u / q, u\right)$ for all sufficiently small $u \in(0, q)$ [using $R\left(x_{1}, 0,0\right)=R\left(x_{2}, 0,0\right)$ and the continuity of $D_{u} R(x, u / q, u)$ in $u$ ]. This proves the lemma.

Proof of TheOREM 2.1. Suppose $0<\kappa<q$. Since $\mathbf{r}(k)=(\kappa / q)^{k}+$ $R(k, \kappa / q, \kappa)$ for $k \in \mathbb{N}$ [see (2.3)] and $\mathbf{r}(x)=R(x, \kappa / q, \kappa)$ for $x \in(-1,0$ ], assertion (2.5) follows directly from Proposition 4.1. Moreover, we infer from the previous lemma that $\mathbf{r}$ is not a constant for infinitely many, in fact all sufficiently small $\kappa \in(0, q)$. We have thus proved the theorem. 
5. Proof of Theorem 2.2. We begin with an auxiliary lemma which gives an asymptotic estimate of the expected extinction time $a_{k}=E_{k} \tau$ as $k \rightarrow \infty$.

LEMMA 5.1. Let $\left(Z_{n}\right)_{n \geq 0}$ be a subcritical GWP with reproduction mean $\mu>0$ and $E Z_{1} \log Z_{1}<\infty$. Then

$$
\lim _{k \rightarrow \infty} \frac{a_{k}}{\log _{1 / \mu} k}=1
$$

PROOF. Recall from [3], Section 1.11, that $f_{n}(0)=1-c_{n} \mu^{n}$ with positive constants $c_{n} \in[0,1]$ converging to some $c>0$. It is the positivity of $c$ where the $(X \log X)$-condition enters. Since $P_{k}(\tau>n)=P_{k}\left(Z_{n}>0\right)=1-f_{n}^{k}(0)$, we infer

$$
\begin{aligned}
\frac{a_{k}}{\log _{1 / \mu} k} & =\frac{1}{\log _{1 / \mu} k} \sum_{n \geq 0} P_{k}(\tau>n) \\
& =\frac{1}{\log _{1 / \mu} k} \sum_{n \geq 0}\left(1-f_{n}^{k}(0)\right) \\
& =\frac{1}{\log _{1 / \mu} k} \sum_{n \geq 0}\left(1-\left(1-c_{n} \mu^{n}\right)^{k}\right) .
\end{aligned}
$$

Fix any $\varepsilon \in(0,1)$, put $n_{*}=n_{*}(\varepsilon, k) \stackrel{\text { def }}{=}(1-\varepsilon) \log _{1 / \mu} k, n^{*}=n^{*}(\varepsilon, k) \stackrel{\text { def }}{=}(1+$ $\varepsilon) \log _{1 / \mu} k$ and split up the sum into three parts, $S_{1}(k), S_{2}(k)$ and $S_{3}(k)$, ranging from 0 to $n_{*}-1$, from $n_{*}$ to $n^{*}-1$ and from $n^{*}$ to $\infty$, respectively. Note that $\mu^{n_{*}}=k^{-(1-\varepsilon)}$ and $\mu^{n^{*}}=k^{-(1+\varepsilon)}$. The three sums will be considered separately.

Choose $m$ such that $\inf _{n \geq m} c_{n} \geq c / 2$. Then we have, for $S_{1}(k)$,

$$
\begin{aligned}
(1- & \left.\varepsilon-\frac{m}{\log _{1 / \mu} k}\right)\left(1-\left(1-c \mu^{m} / 2\right)^{k}\right) \\
& \leq \frac{1}{\log _{1 / \mu} k} \sum_{n=m}^{n_{*}-1}\left(1-\left(1-c \mu^{n} / 2\right)^{k}\right) \leq S_{1}(k) \\
& \leq \frac{1}{\log _{1 / \mu} k} \sum_{n=0}^{n_{*}-1}\left(1-\left(1-\mu^{n}\right)^{k}\right) \leq(1-\varepsilon)\left(1-\left(1-\mu^{n_{*}}\right)^{k}\right) \\
& =(1-\varepsilon)\left(1-\left(1-k^{-(1-\varepsilon)}\right)^{k}\right) \leq(1-\varepsilon)\left(1-\exp \left(-2 k^{\varepsilon}\right)\right),
\end{aligned}
$$

where the last inequality holds for sufficiently large $k$ using $\log (1-x) \geq-2 x$ for all positive $x$ sufficiently close to 0 . Consequently,

$$
\lim _{k \rightarrow \infty} S_{1}(k)=1-\varepsilon .
$$


For $S_{2}(k)$ we just note that $0 \leq S_{2}(k) \leq 2 \varepsilon$. Finally, we obtain for $S_{3}(k)$, if $k$ is sufficiently large,

$$
\begin{aligned}
0 \leq S_{3}(k) & \leq \frac{1}{\log _{1 / \mu} k} \sum_{n \geq 0}\left(1-\left(1-\mu^{n^{*}+n}\right)^{k}\right) \\
& =\frac{1}{\log _{1 / \mu} k} \sum_{n \geq 0}\left(1-\left(1-k^{-(1+\varepsilon)} \mu^{n}\right)^{k}\right) \\
& \leq \frac{1}{\log _{1 / \mu} k} \sum_{n \geq 0}\left(1-\exp \left(-2 k^{-\varepsilon} \mu^{n}\right)\right) \\
& \leq \frac{2}{k^{\varepsilon} \log _{1 / \mu} k} \sum_{n \geq 0} \mu^{n},
\end{aligned}
$$

where $1-e^{-x} \leq x$ for all $x$ has been used for the final inequality. Hence,

$$
\lim _{k \rightarrow \infty} S_{3}(k)=0 .
$$

Putting the results together, the assertion of the lemma easily follows because $\varepsilon \in(0,1)$ was arbitrary.

ProOF OF THEOREM 2.2. We first note that

$$
\frac{\mathbf{q}(k)}{a_{k} q^{k}}=\frac{\mathbf{r}(k)}{a_{k}}=\frac{1}{a_{k}}\left(1+E_{k}\left(\sum_{j=1}^{\tau} \prod_{i=0}^{j-1}\left(1-q^{Z_{i}}\right)\right)\right)
$$

because $\kappa=q$. We thus have to show

$$
\lim _{k \rightarrow \infty} \frac{1}{a_{k}} E_{k}\left(\sum_{j=1}^{\tau}\left(1-\prod_{i=0}^{j-1}\left(1-q^{Z_{i}}\right)\right)\right)=0 .
$$

Fix an arbitrary $\varepsilon \in(0,1)$, put $N(k) \stackrel{\text { def }}{=}\left\lfloor\varepsilon a_{k}\right\rfloor$ and split up the sum under the expectation into the sum from 1 to $\tau-N(k)-1$ [of course, equal to 0 if $\tau-N(k) \leq 0]$ and the sum from $\tau-N(k)$ to $\tau$. As for the latter, we immediately have

$$
0 \leq \limsup _{k \rightarrow \infty} \frac{1}{a_{k}} E_{k}\left(\sum_{j=\tau-N(k)}^{\tau}\left(1-\prod_{i=0}^{j-1}\left(1-q^{Z_{i}}\right)\right)\right) \leq \lim _{k \rightarrow \infty} \frac{N(k)+1}{a_{k}}=\varepsilon .
$$

Turning to the first sum, we use once more the inequality $1-\prod_{i=0}^{n}\left(1-x_{i}\right) \leq$ $\sum_{i=0}^{n} x_{i}$ for numbers $x_{1}, \ldots, x_{n} \in[0,1]$ and obtain [with $\left(Z_{n}^{\prime}\right)_{n \geq 0}$ as in (4.4)]

$$
\begin{aligned}
& \frac{1}{a_{k}} E_{k}\left(\sum_{j=1}^{\tau-N(k)-1}\left(1-\prod_{i=0}^{j-1}\left(1-q^{Z_{i}}\right)\right)\right) \\
& \quad \leq \frac{1}{a_{k}} E_{k}\left(\sum_{j=1}^{\tau-N(k)-1} \sum_{i=0}^{j-1} q^{Z_{i}}\right) \leq \frac{1}{a_{k}} E_{k}\left(\sum_{j \geq 1} \sum_{i=0}^{j-1} q^{Z_{i}} \mathbb{1}_{\left\{Z_{j+N(k)}>0\right\}}\right)
\end{aligned}
$$




$$
\begin{aligned}
& =\frac{1}{a_{k}} \sum_{i \geq 0} \sum_{j>i} E_{k}\left(q^{Z_{i}} P_{Z_{i}}\left(Z_{j-i+N(k)}^{\prime}>0\right)\right) \\
& =\frac{1}{a_{k}} \sum_{i \geq 0} \sum_{j>N(k)} E_{k}\left(q^{Z_{i}}\left(1-f_{j}^{Z_{i}}(0)\right)\right) \\
& =\frac{1}{a_{k}} \sum_{i \geq 0} \sum_{j>N(k)}\left(f_{i}^{k}(q)-f_{i}^{k}\left(q f_{j}(0)\right)\right) .
\end{aligned}
$$

Now a first-order Taylor expansion of $f_{i}^{k}\left(q f_{j}(0)\right)$ about $q$ together with the monotonicity of $f_{i}$ and $f_{i}^{\prime}$ gives, for some $z_{i j}$ between $q f_{j}(0)$ and $q$,

$$
\begin{aligned}
f_{i}^{k}\left(q f_{j}(0)\right) & =f_{i}^{k}(q)-k q f_{i}^{k-1}\left(z_{i j}\right) f_{i}^{\prime}\left(z_{i j}\right)\left(1-f_{j}(0)\right) \\
& \geq f_{i}^{k}(q)-k q f_{i}^{k-1}(q) f_{i}^{\prime}(1)\left(1-f_{j}(0)\right) .
\end{aligned}
$$

Hence, the above estimation can be continued as

$$
\begin{aligned}
& \leq \frac{k q}{a_{k}}\left(\sum_{i \geq 0} f_{i}^{k-1}(0) f_{i}^{\prime}(1)\right)\left(\sum_{j>N(k)}\left(1-f_{j}(0)\right)\right) \\
& \leq \frac{k q}{a_{k}}\left(\sum_{i \geq 0} f_{i}^{k-1}(q) \mu^{i}\right)\left(\sum_{j>N(k)} 2 c \mu^{j}\right) .
\end{aligned}
$$

Now the second sum in (5.1) is clearly bounded by a constant times $\mu^{\varepsilon \log _{1 / \mu} k}=$ $k^{-\varepsilon}$ for all $k$ [since $N(k)=\left\lfloor\varepsilon a_{k}\right\rfloor \simeq \varepsilon \log _{1 / \mu} k$ by Lemma 5.1], while the first can be bounded by a constant times $k^{-(1-\varepsilon)}$ for sufficiently large $k$. To see the latter, split up the first sum as

$$
\left(\sum_{i=0}^{\left\lfloor(1-\varepsilon) \log _{1 / \mu} k\right\rfloor}+\sum_{i>\left\lfloor(1-\varepsilon) \log _{1 / \mu} k\right\rfloor}\right) f_{i}^{k-1}(q) \mu^{i} .
$$

Observe that

$$
\sum_{i>\left\lfloor(1-\varepsilon) \log _{1 / \mu} k\right\rfloor} f_{i}^{k-1}(q) \mu^{i} \leq \frac{\mu^{(1-\varepsilon) \log _{1 / \mu} k}}{1-\mu}=\frac{k^{-(1-\varepsilon)}}{1-\mu} .
$$

Since, for all $i_{0} \leq i \leq(1-\varepsilon) \log _{1 / \mu} k, i_{0}$ sufficiently large (independent of $k$ ), all $k$ sufficiently large and some $Q(q) \in(-1,0)$ (see [3], Section 1.11),

$$
\begin{aligned}
f_{i}^{k-1}(q) & \leq\left(1+Q(q) \mu^{i} / 2\right)^{k-1} \\
& \leq\left(1+Q(q) \mu^{(1-\varepsilon) \log _{1 / \mu} k} / 2\right)^{k-1} \leq \exp \left((k-1)^{\varepsilon} Q(q) / 2\right),
\end{aligned}
$$

we further have 


$$
\sum_{i=0}^{\left\lfloor(1-\varepsilon) \log _{1 / \mu} k\right\rfloor} f_{i}^{k-1}(q) \mu^{i} \leq i_{0} f_{i_{0}}^{k-1}(q)+(1-\varepsilon) \log _{1 / \mu} k \exp \left((k-1)^{\varepsilon} Q(q) / 2\right)
$$

for all $k$ sufficiently large.

Putting the pieces together, we finally conclude in (5.1) that

$$
\frac{k q}{a_{k}}\left(\sum_{i \geq 0} f_{i}^{k-1}(q) \mu^{i}\right)\left(\sum_{j>N(k)} 2 c \mu^{j}\right) \leq \frac{\text { const }}{a_{k}},
$$

which converges to 0 as $k \rightarrow \infty$.

\section{REFERENCES}

[1] Alsmeyer, G. and Rösler, U. (1996). The bisexual Galton-Watson process with promiscuous mating: extinction probabilities in the supercritical case. Ann. Appl. Probab. 6922 939.

[2] Alsmeyer, G. and Rösler, U. (2001). The entrance Martin boundary of the Galton-Watson process. Unpublished manuscript.

[3] Athreya, K. B. and Ney, P. (1972). Branching Processes. Springer, New York.

[4] Biggins, J. D. and NAdARAJAH, S. (1993). Near-constancy of the Harris function in the simple branching process. Comm. Statist. Stochastic Models 9 435-444.

[5] BRuss, T. (1984). A note on extinction criteria for bisexual Galton-Watson processes. J. Appl. Probab. 21 915-919.

[6] Daley, D. (1968). Extinction probabilities for certain bisexual Galton-Watson branching processes. Z. Wahrsch. Verw. Gebiete 9 315-322.

[7] Daley, D., Hull, D. M. and Taylor, J. M. (1986). Bisexual Galton-Watson branching processes with superadditive mating functions. J. Appl. Probab. 23 585-600.

[8] Hull, D. M. (1982). A necessary condition for extinction in those bisexual Galton-Watson branching processes governed by superadditive mating functions. J. Appl. Probab. 19 $847-850$.

[9] Hull, D. M. (1984). Conditions for extinction in certain bisexual Galton-Watson branching processes. J. Appl. Probab. 21 414-418.

INSTITUT FÜR MATHEMATISCHE STATISTIK

FACHBEREICH MATHEMATIK

WESTFÄLISCHE WILHELMS-UNIVERSITÄT

MÜNSTER

EINSTEINSTRASSE 62

D-48149 MÜNSTER

GERMANY

E-MAIL: gerolda@math.uni-muenster.de
MATHEMATISCHES SEMINAR

Christian-Albrechts-Universität KIEL

LUDEWIG-MEYN-STRASSE 4

D-24098 KIEL

GERMANY

E-MAIL: roesler@math.uni-kiel.de 